\title{
Implant placement with guided bone regeneration in esthetic zone- A case report
}

\author{
Maharjan $\mathrm{A}^{1}$, Joshi $\mathrm{S}^{2}$, Verma $\mathrm{A}^{3}$, Gorkhali $\mathrm{RS}^{4}$ \\ ${ }^{1}$ Consultant Prosthodontist, Bharatpur Government Hospital, Chitwan, Nepal \\ ${ }^{2}$ Professor, Department of Prosthodontics, National Academy of Medical Sciences, Kathmandu, Nepal \\ ${ }^{3}$ Assistant Professor, Department of Prosthodontics, National Academy of Medical Sciences, Kathmandu, Nepal. \\ ${ }^{4}$ Assistant Professor, Department of Periodontology and Oral Implantology, National Academy of Medical Sciences, \\ Kathmandu, Nepal.
}

\begin{abstract}
Alveolar ridge deficiency is considered a major limitation for successful implant placement, as well as for the long-term success rate, especially in the anterior maxillary region. Implants placed without regard for prosthetic position often results in dental restorations that are functionally and esthetically compromised. Adequate peri-implant bone support is essential for immediate and long-term implant stability, as well as for future esthetic outcome. To achieve this goal, augmentation of lost bone is often necessary.

A variety of surgical approaches have been proposed to enhance the alveolar bone volume. Guided bone regeneration (GBR) is the most common technique for localized bone augmentation. GBR, by application of cell occlusive membranes that mechanically exclude non-osteogenic cell populations from the surrounding soft tissues, has become a well-documented and highly successful procedure for localized augmentation of the atrophic jaw before or simultaneously with implant placement.

This case report presents simultaneous approach of guided bone regeneration and implant placement in the maxillary anterior region with narrow ridge defect.
\end{abstract}

Key words: Augmentation, Esthetic outcome, Guided Bone Regeneration, Implant, Maxillary Anterior

\section{Introduction}

$\mathrm{I}$ mplant therapy in the anterior maxilla is challenging for the clinician because of esthetic demand of the patients and difficult preexisting anatomy. ${ }^{1}$ In these cases, clinicians are often confronted with tissue deficiencies caused by various conditions. The bony housing in this instance would require augmentation to provide a configuration that permits placement of implants in optimal positions which in turn will result in pleasing aesthetics. Various

\section{Conflict of Interest: No}

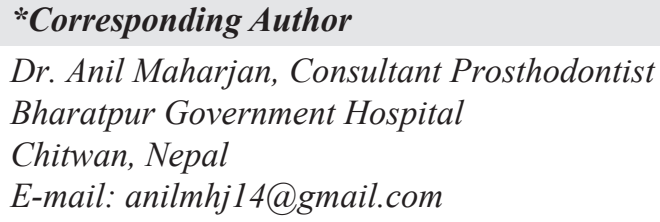

surgical techniques have been proposed in the past 15 years to correct such bony defects at the facial aspect of potential implant sites. This includes onlay grafting, GBR using barrier membranes, ${ }^{2,3}$ a combination of block bone grafts and barrier membranes, and distraction osteogenesis. Careful soft tissue handling, precise implant placement in a prosthetically driven 3D approach and follow-up procedures represent a variety of challenges for the implant surgeon. This clinical report presents implant placement in maxillary esthetic zone with deficient buccal bone using GBR.

\section{Clinical case}

A 38 years old male patient came to the Department of Prosthodontics, National Academy of Medical Sciences, Bir Hospital with the chief complaint of missing tooth in 
upper front region since one year. Patient had lost the tooth because of caries and wanted replacement for esthetic reasons. The prosthetic options were discussed and patient opted implant as a treatment modality. Extraoral and intraoral examinations were done (Figure 1, $2)$. Gingival biotype in this area was thick and there was concavity or crater like defect with deficient buccal bone, Siebert's class I defect (Figure 2). Primary impressions were made with alginate for study models. Buccolingual and mesiodistal dimensions in edentulous area in respect to 12 were measured. Patient was advised for Cone Beam Computed Tomography (CBCT), buccolingual width was measured $4.67 \mathrm{~mm}$ and there was adequate height for implant placement (Figure 3). As implant had to be placed in anterior maxilla, esthetic risk analysis was done. The factors that contributed to low risk was healthy patient, nonsmoker with thick gingival biotype. The factors that contributed to medium risk included patient expectation, bone level at adjacent teeth was about $5.5 \mathrm{~mm}$ to contact point and horizontal bone deficiency present. Implant planning was done and implant size selected was $3 \times 10 \mathrm{~mm}$ (Dio Implant System).

Patient was prepared for the surgery. Local anaesthetic infiltration was given (2\% xylocaine, 1:80000). Crestal incision was made $2 \mathrm{~mm}$ towards the palatal aspect that extended through the sulcus of adjacent teeth to reflect full thickness mucoperiosteal flap. Osteotomy was started with the help of surgical template used as a guide made from vacuum formed sheet (Figure 4). Implant was placed considering buccolingual, mesiodistal and apicocoronal position (Figure 5). There was thin facial bone buccal to the implant that might undergo resorption in future (Figure 6). Therefore, contour augmentation was done to achieve long term treatment outcome.

For GBR, xenograft (Biooss 0.5g) and collagen membrane (Biogide membrane $13 \times 25 \mathrm{~mm}$ ) were used (Figure 7). Stabilization of the membrane and the underlying graft material was achieved by using horizontal mattress sutures extending from the apical portion of the facial periosteum to the palatal aspect of the flap. Primary closure with Polydioxanone II 4.0 Suture (Ethicon) was done and was allowed to heal. After 6 months, second stage surgery was performed to gain access to the implant site. Gingival former of $4.2 \mathrm{~mm}$ diameter, $2 \mathrm{~mm}$ cuff height was placed (Figure 8). After 2 weeks, impression was made with polyvinyl siloxane putty and light body using single step closed tray impression technique (Figure 9) and sent to dental laboratory for fabrication of multilayered zirconia crown. The crown was cemented to the abutment with resin modified Glass Ionomer Cement (GIC) (Figure 10). Excess cement was removed and occlusion was verified. An intraoral periapical radiograph was taken (Figure 11). Oral hygiene instructions were reinforced and recalled for regular follow-ups.
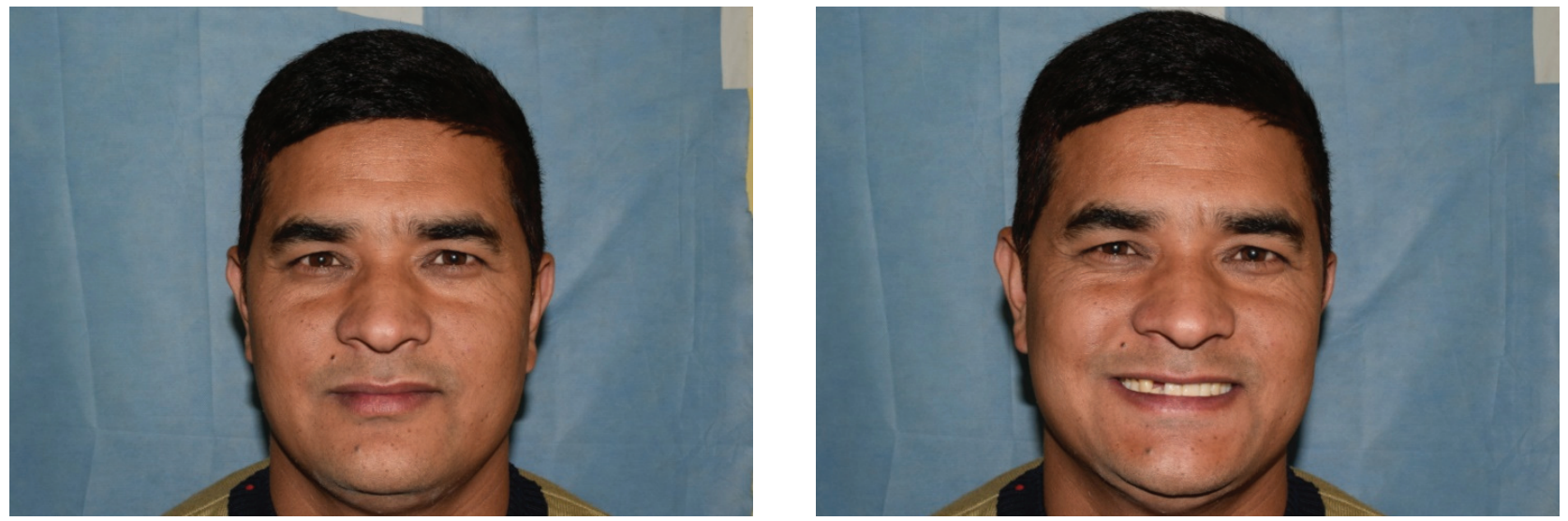

Figure 1: Extraoral Photographs 

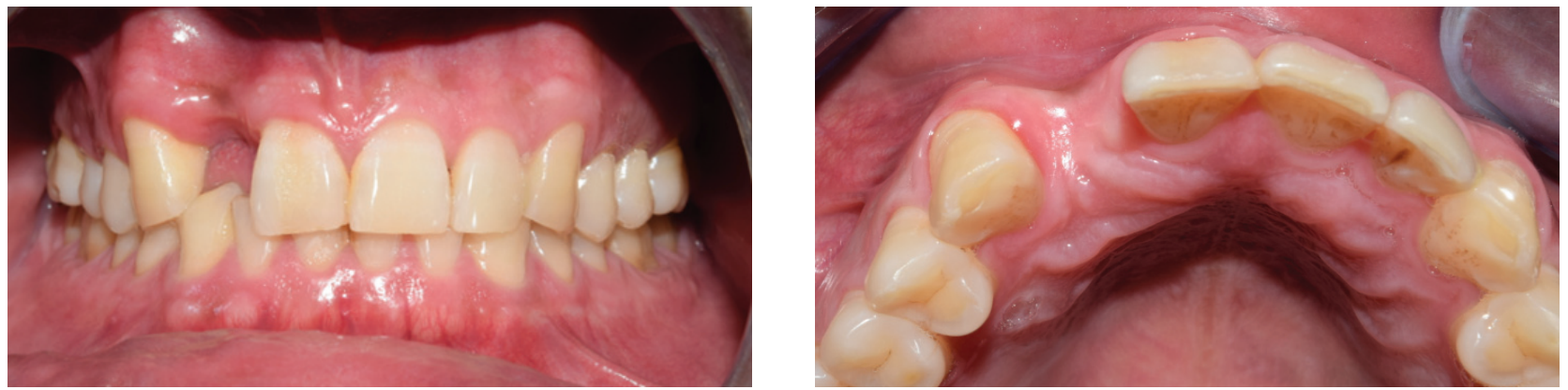

Figure 2: Intraoral Photographs
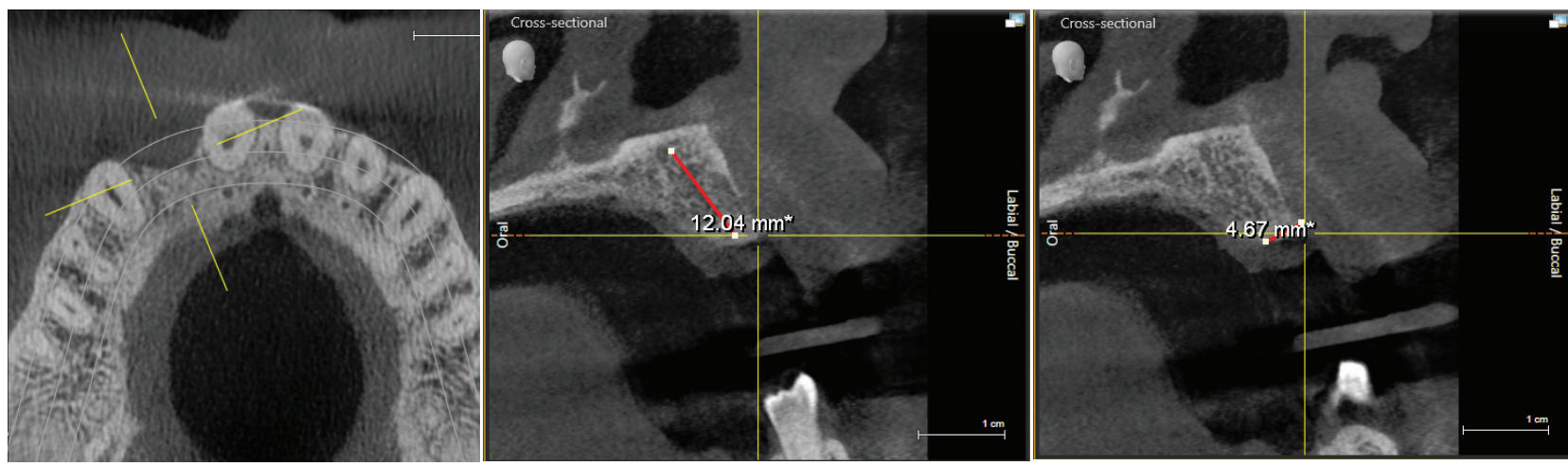

Figure 3: CBCT Images i.r.t 12

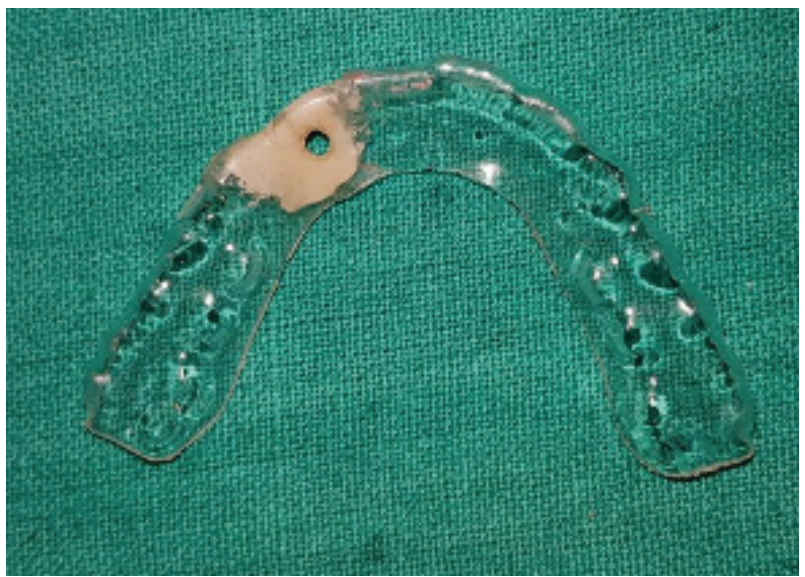

Figure 4: Surgical Guide
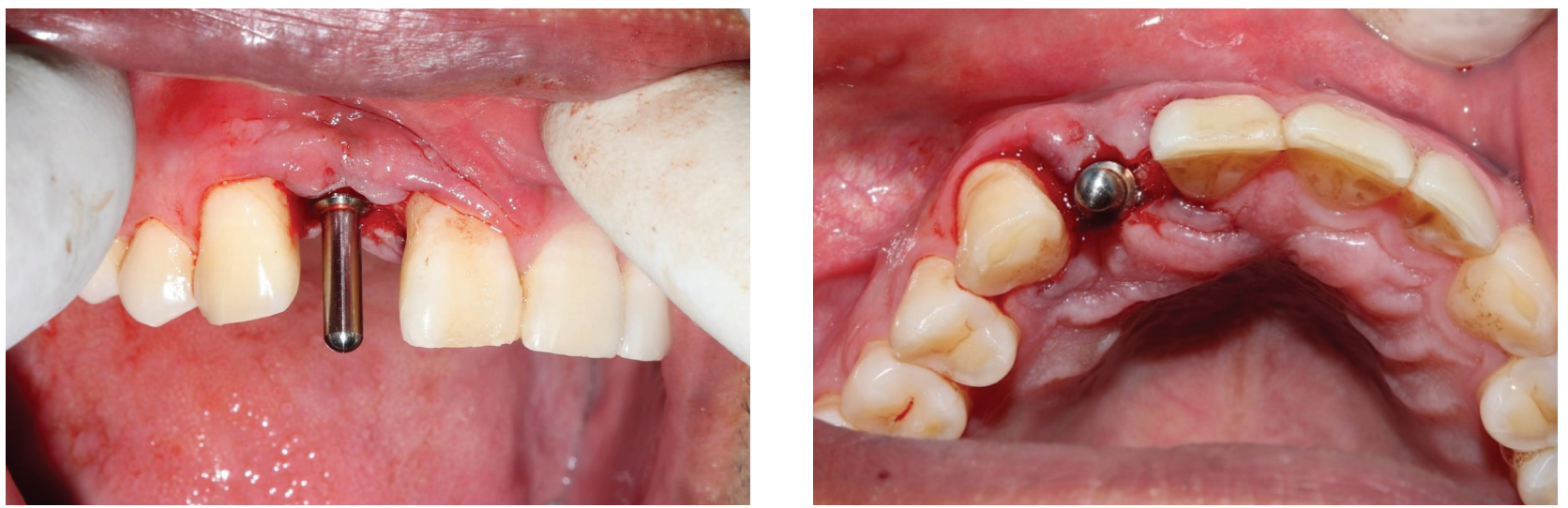

Figure 5: Parallel Pin in Place 


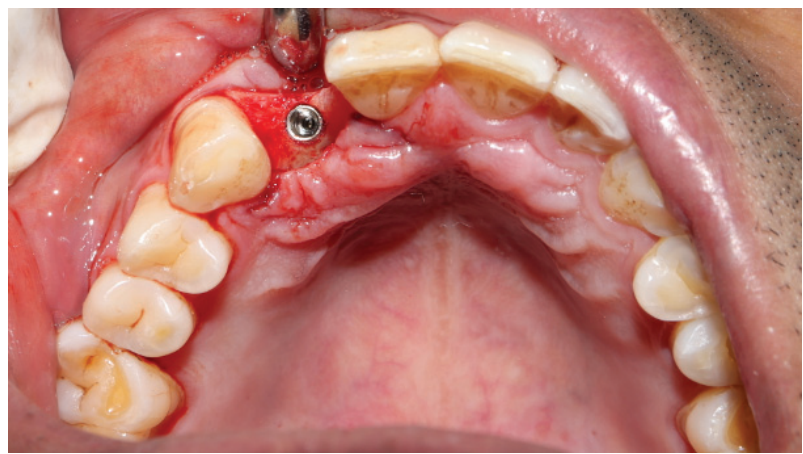

Figure 6: Dio Implant $3.0 \mathrm{~mm} \times 10 \mathrm{~mm}$

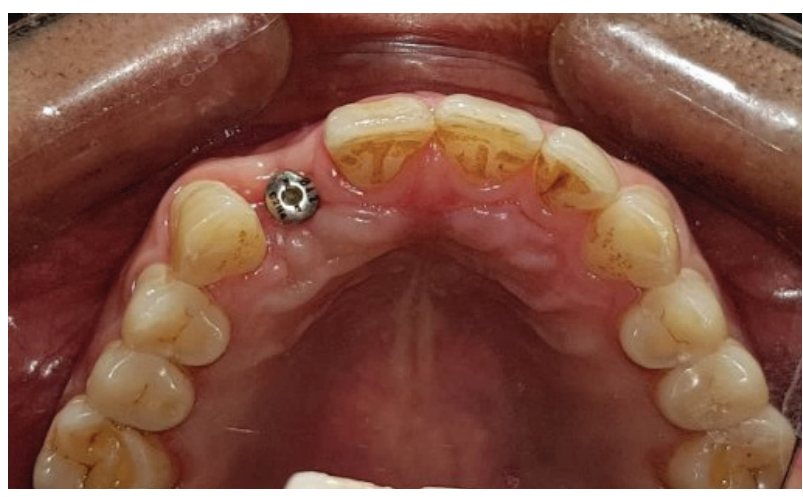

Figure 8: Healing abutment- $4.2 \times 2 \mathrm{~mm}$

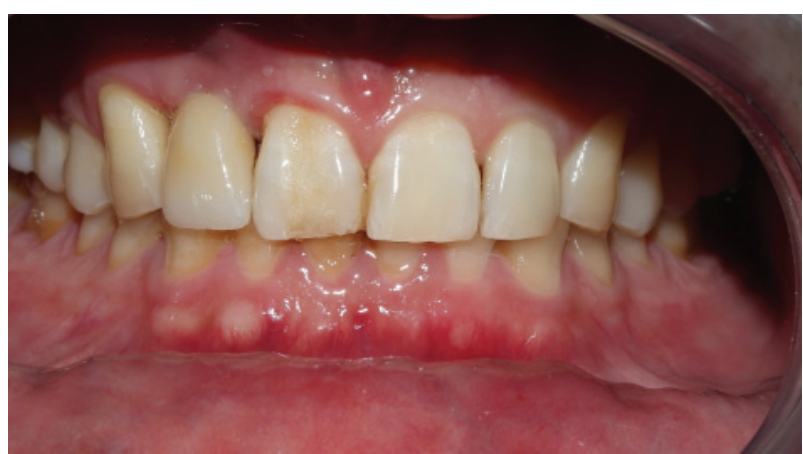

Figure 10: Final Prosthesis Placement

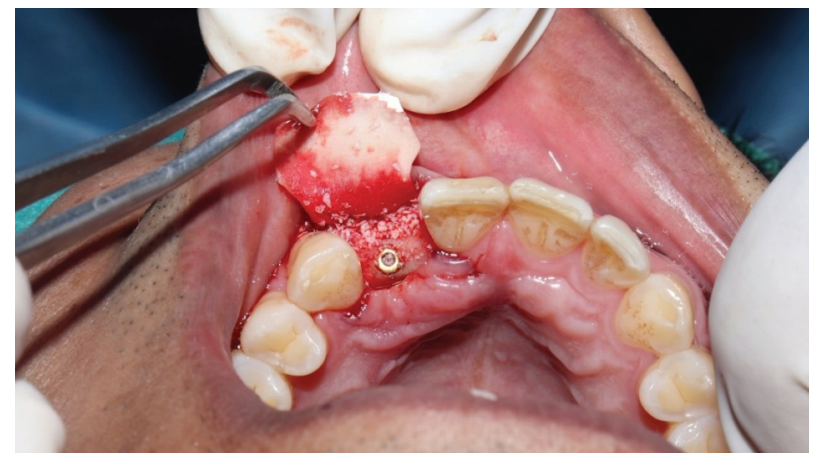

Figure 7: Bio-oss particles and bio-gide membrane in place

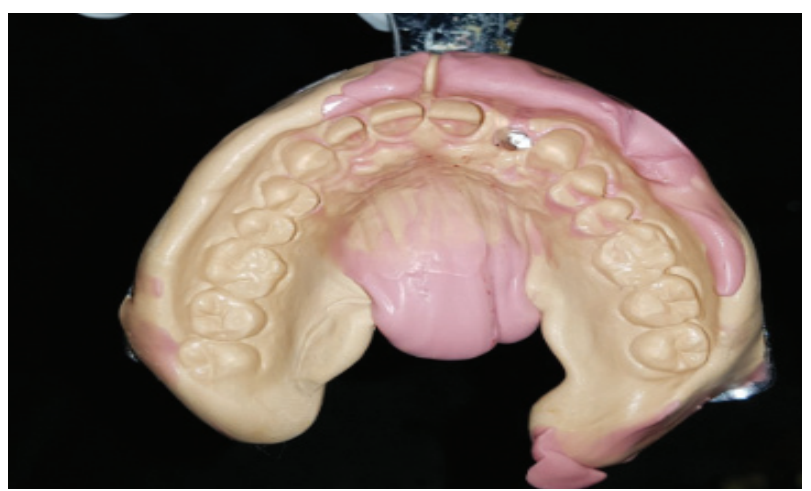

Figure 9: Putty wash closed tray impression

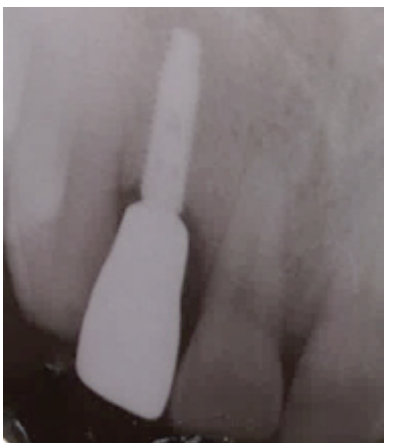

Figure 11: Postoperative IOPAR-12

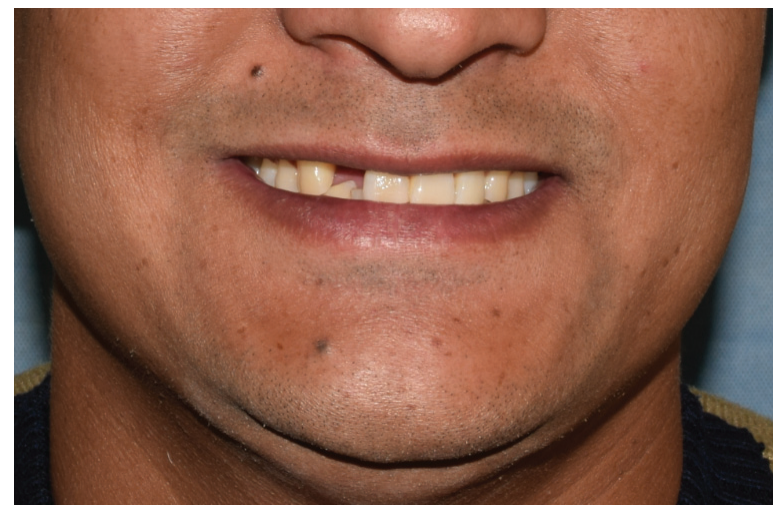

Preoperative Photograph 

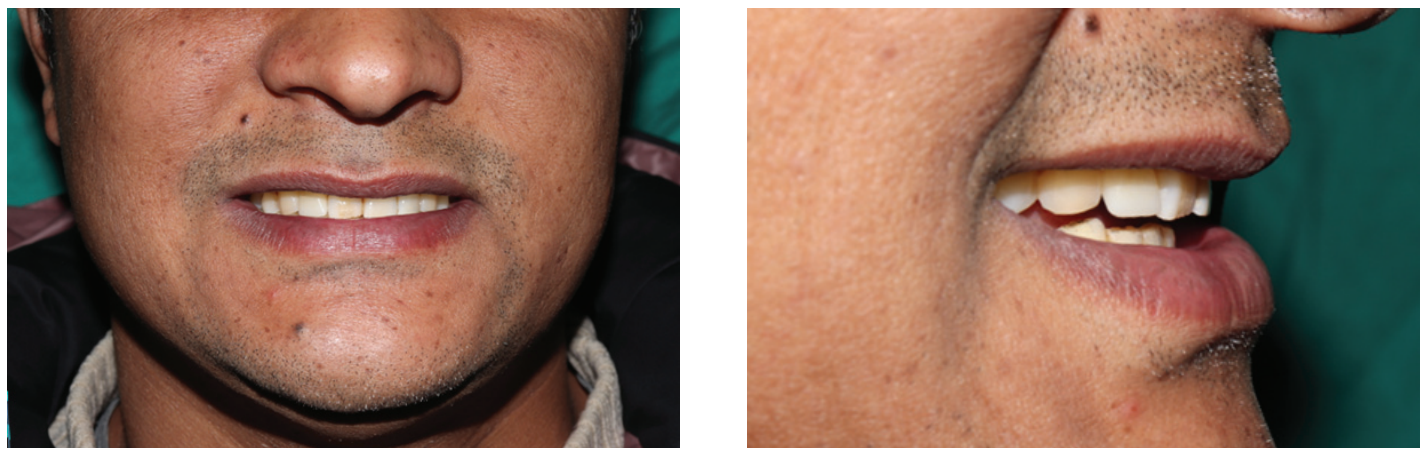

Postoperative Photographs

\section{Discussion}

For bone augmentation, there are various surgical techniques which include onlay or veneer grafts, ridge splitting, bone condensation and GBR. Guided Bone Regeneration (GBR) is a technique in which bone growth is enhanced by maintaining the space and preventing soft tissue growth into the area utilizing either a resorbable or non-resorbable barrier membrane and achieving the bone regeneration. It may be performed in conjunction with the placement of the implant or during a surgical intervention prior to implant placement. From a scientific point of view, the GBR technique is a welldocumented procedure that can be used with either a simultaneous or a staged approach., Clinical studies demonstrate that horizontal bone augmentation can be predictably obtained with the GBR technique. ${ }^{7}$ A study done by Jung et al found that implants placed simultaneously with GBR procedures using resorbable or nonresorbable membranes reveal a high survival rate ranging from $91.9 \%$ to $92.6 \%$, therefore it is considered to be a safe and predictable therapy. ${ }^{8}$

Various different membrane materials have been used in experimental and clinical studies in the context of GBR treatment. In this case, absorbable collagen membrane was used. The advantage of using absorbable membrane is that a second procedure to remove the membrane is not necessary. The membrane is made with a unique manufacturing process which creates a longer resorption profile suited for GBR procedures. In this case, as the width of bone defect was less than $5 \mathrm{~mm}$ with crater like osseous defect on the buccal bone, GBR simultaneous approach was chosen. ${ }^{9}$ Bio-oss is a natural, osteoconductive bone substitute with effective and predictable bone regeneration. ${ }^{10}$

Buser $^{1}$ has highlighted different anatomical factors to be analysed in the anterior maxilla which includes location of smile line, gingival morphotype, interocclusal relationship, dimensions of edentulous gap, anatomy of alveolar crest, status of adjacent dentition, radiographic status. These all factors should be considered during treatment planning in maxillary anterior region.

Esthetic failures can also be caused by inappropriate implant positioning and/or improper implant selection. Placement of implants in a correct 3-dimensional position is key to an esthetic treatment outcome regardless of the implant system used. The relationship of the position between the implant and the proposed restoration should be based on the position of the implant, because this will influence the final hard and soft tissue response. The implant position can be viewed in 3 dimensions: buccolingual, mesiodistal, and apicocoronal. An implant placed too far facially will result in a potential risk for soft tissue recession, because the thickness of the facial bone wall is clearly reduced by the malpositioned implant. In addition, potential prosthetic complications could result in restoration-implant axis 
problems, making the implant difficult to restore. Implants positioned too far palatally can result in emergence problems, as seen with ridge-lap restorations. These restorations can be unesthetic and extremely difficult to maintain, and should therefore be avoided. ${ }^{11}$ Improper mesiodistal positioning of implants can have a substantial effect on the interproximal papillary support as well as on the osseous crest on the adjacent natural tooth. Apicocoronally, implant shoulder should be approximately $2 \mathrm{~mm}$ apical to the midfacial gingival margin of the planned restoration. This can be accomplished through the use of surgical templates that highlight the gingival margin of the planned restoration. These all factors should be considered during planning of implant placement in maxillary anterior region.

\section{Conclusion}

To maximize functional and esthetic results, implants should be placed according to prosthetic needs and design. Due to bone resorption after extraction, ideal placement of implants would be often impossible without prior hard and soft tissue augmentation. Several techniques such as GBR, onlay veneer grafting, inter-positional inlay grafting, ridge splitting technique and distraction osteogenesis are available nowadays. Based on advantages and disadvantages of these techniques, a single or a combination of these techniques can be used. Thus, a deep knowledge of these techniques will allow the surgeon to properly select the right combination for prosthetic needs and especially for the esthetic and function of patients.

\section{References}

1. Buser D, Martin W, Belser UC. Optimizing esthetics for implant restorations in the anterior maxilla: anatomic and surgical considerations. Int J Oral Maxillofac Implants. 2004.

2. Nyman S, Lang NP, Buser D, Brägger U. Bone regeneration adjacent to titanium dental implants using guided tissue regeneration: a report of two cases. Int J Oral Maxillofac Implants.1990.

3. Buser D, Brägger U, Lang NP, Nyman S. Regeneration and enlargement of jaw bone using guided tissue regeneration. Clin Oral Implants Res. 1990;1(1):22-32.

4. Bister D. The SAC classification in implant dentistry. $1^{\text {st }}$ Ed. QP Deutschland; 2009.

5. Buser D, Dahlin C, Schenk RK. Guided bone regeneration. Chicago Quintessence. 1994.

6. Nyman S, Lang NP, Buser D, Brägger U. Bone regeneration adjacent to titanium dental implants using guided tissue regeneration: a report of two cases. Int J Oral Maxillofac Implants. 1990. $1 ; 5(1)$

7. Buser D, Dula K, Hirt HP, Schenk RK. Lateral ridge augmentation using autografts and barrier membranes: a clinical study with 40 partially edentulous patients. J Oral Maxillofac Surg. 1996 Apr 1;54(4):420-32.

8. Jung RE, Fenner N, Hämmerle $\mathrm{CH}$, Zitzmann NU. Long-term outcome of implants placed with guided bone regeneration (GBR) using resorbable and non-resorbable membranes after 12-14 years. Clin oral Implants Res. 2013 Oct;24(10):1065-73.

9. Sato N. Periodontal surgery: a clinical atlas.

10. Aghaloo TL, Moy PK. Which hard tissue augmentation techniques are the most successful in furnishing bony support for implant placement?. Int J Oral Maxillofac Implants. 2007. 2;22(7).

11. Belser UC, Bernard JP, Buser D. Implantsupported restorations in the anterior region: prosthetic considerations. Prac Periodontics Aesthet Dent.1996.1;8(9):875-3. 